\title{
Coarse graining and decoherence in quantum field theory
}

\author{
Fernando Lombardo* \\ Departamento de Física, Facultad de Ciencias Exactas y Naturales \\ Universidad de Buenos Aires- Ciudad Universitaria, Pabellón I \\ 1428 Buenos Aires, Argentina \\ Francisco D. Mazzitelli ${ }^{\dagger}$ \\ Departamento de Física, Facultad de Ciencias Exactas y Naturales \\ Universidad de Buenos Aires- Ciudad Universitaria, Pabellón I \\ 1428 Buenos Aires, Argentina \\ and \\ Instituto de Astronomía y Física del Espacio \\ Casilla de Correo 67-Sucursal 28 \\ 1428 Buenos Aires, Argentina
}

\begin{abstract}
We consider a $\lambda \phi^{4}$ theory in Minkowski spacetime. We compute a "coarse grained effective action" by integrating out the field modes with wavelength shorter than a critical value. From this effective action we obtain the evolution equation for the reduced density matrix (master equation). We compute the diffusion coefficients of this equation and analyze the decoherence induced on the long- wavelength modes. We generalize the results to the case of a conformally coupled scalar field in DeSitter spacetime. We show that the
\end{abstract}

\footnotetext{
*e-mail: lombardo@df.uba.ar

${ }^{\dagger}$ e-mail: fmazzi@df.uba.ar
} 
decoherence is effective as long as the critical wavelength is taken to be not shorter than the Hubble radius.

PACS numbers: 03.70.+k, 05.40.+j 


\section{INTRODUCTION}

The quantum to classical transition [1] is a very old and interesting problem relevant in many branches of physics. It involves the concepts of correlations, i.e. the Wigner function of the quantum system should have a peak at the classical trajectories, and decoherence, that is, there should be no interference between classical trajectories. The density matrix should be diagonal. In order to understand the emergence of classical behavior, it is essential to consider the interaction of the system with its environment [2], since both the loss of quantum coherence and the onset of classical correlations depend strongly on this interaction [3, 4 ,

In the last years, there has been a renewed interest in the study of this transition in the context of quantum cosmology [5]. The efforts are directed to explain the emergence of a classical space-time metric from a full quantum theory, and to derive the Einstein equations, or the quantum-corrected version of them. The relevance of statistical and quantum effects such as noise, dissipation, particle creation, back-reaction, etc, have been subsequently elucidated in the context of quantum Brownian motion (QBM) [6] and quantum field theory [7].

The quantum to classical transition is also of interest in the standard cosmology. According to the inflationary theory, quantum fluctuations of matter fields are the origin of very small inhomogeneities which gave rise to the formation of structures in the Universe [10]. A crucial assumption in this scenario is that quantum fluctuations become classical as their wavelength become larger than the Hubble radius $H^{-1}$. Therefore, a detailed analysis of this classical to quantum transition is needed in order to complete our understanding of the mechanism of structure-formation.

There has been several attempts in order to prove the previously mentioned assumption. In Ref. [11], this problem has been analyzed using a toy model which consists of two

massless fields interacting through a mixing of the kinetic terms. Similar theories involving interactions with mass mixing terms have been considered in Ref. [12. In all these models, the interaction terms are quadratic and disappear after a redefinition of the fields. In Ref. 
[13], the same problem has been studied in a theory consisting of two scalar fields with a biquadratic interaction.

The aim of this paper is to analyze the emergence of classical inhomogeneities from quantum fluctuations in a more realistic model. To begin with, we will consider a $\lambda \phi^{4}$ theory in Minkowski spacetime. Following Starobinsky's original suggestion for the stochastic inflationary model [14], we will split the scalar field into two parts: the system $\phi_{<}$containing the field modes whose wavelengths are longer than certain fixed cutoff $\left(\Lambda^{-1}\right)$, and the environment $\phi_{>}$which contains the modes with wavelengths shorter than the cutoff. Although this splitting will cause some technical difficulties, we will see that it is possible to study and prove the assertion that quantum fluctuations with wavelengths longer than $\Lambda^{-1}$ decohere due to the interaction with the environment. Moreover, it will be also possible to extend the results to curved spaces. For a conformally coupled scalar field in a DeSitter spacetime, we will show that Starobinsky's splitting between system and environment is the most adequate in the following sense: the minimum value for the critical wavelength such that the system looses coherence is $\Lambda^{-1} \sim H^{-1}$. For shorter critical wavelengths there are modes in the system that do not decohere.

The paper is organized as follows. In the next Section we review the relation between the reduced density matrix for the system and the Feynman-Vernon influence functional [13,15]. We integrate the environment degrees of freedom and compute this functional perturbatively in $\lambda$, for an arbitrary critical wavelength $\Lambda^{-1}$. In Section 3 , we compute the propagator for the reduced density matrix and indicate the procedure to obtain the master equation. In particular we compute the diffusion coefficients, since they give the main contribution to the decoherence. Section 4 contains the analysis of the loss of coherence produced by each diffusion coefficient, with particular emphasis on the cutoff-dependence of the results. We also extend the results to the case of a conformally coupled scalar field in DeSitter spacetime, and discuss whether the choice $\Lambda \sim H$ is relevant or not. Section 5 contains our final remarks. 


\section{THE INFLUENCE FUNCTIONAL AND THE DENSITY MATRIX}

Let us consider a quantum scalar field in a Minkowski spacetime with a quartic selfinteraction. The classical action is given by

$$
S[\phi]=\int d^{4} x\left\{\frac{1}{2} \partial_{\nu} \phi(x) \partial^{\nu} \phi(x)-\frac{1}{2} m^{2} \phi^{2}(x)-\frac{1}{4 !} \lambda \phi^{4}(x)\right\},
$$

where $m$ is the bare mass of the scalar field and $\lambda$ is the bare coupling constant. Let us make a system-environment field splitting

$$
\phi(x)=\phi_{<}(x)+\phi_{>}(x),
$$

where we define the system by

$$
\phi_{<}(\vec{x}, t)=\int_{|\vec{k}|<\Lambda} \frac{d^{3} \vec{k}}{(2 \pi)^{3}} \phi(\vec{k}, t) \exp i \vec{k} \cdot \vec{x},
$$

and the environment by

$$
\phi_{>}(\vec{x}, t)=\int_{|\vec{k}|>\Lambda} \frac{d^{3} \vec{k}}{(2 \pi)^{3}} \phi(\vec{k}, t) \exp i \vec{k} \cdot \vec{x} .
$$

The system-field contains the modes with wavelengths longer than the critical value $\Lambda^{-1}$, while the bath or environment-field contains wavelengths shorter than $\Lambda^{-1}$.

After the splitting, the total action (四) can be written as

$$
S[\phi]=S_{0}\left[\phi_{<}\right]+S_{0}\left[\phi_{>}\right]+S_{\text {int }}\left[\phi_{<}, \phi_{>}\right],
$$

where $S_{0}$ denotes the free field action and the interaction term is given by

$$
\begin{aligned}
S_{\text {int }}\left[\phi_{<}, \phi_{>}\right]=-\int d^{4} x\left\{\frac{\lambda}{4 !} \phi_{<}^{4}(x)\right. & +\frac{\lambda}{4 !} \phi_{>}^{4}(x)+\frac{\lambda}{4} \phi_{<}^{2}(x) \phi_{>}^{2}(x) \\
& \left.+\frac{\lambda}{6} \phi_{<}^{3}(x) \phi_{>}(x)+\frac{\lambda}{6} \phi_{<}(x) \phi_{>}^{3}(x)\right\} .
\end{aligned}
$$

The total density matrix (for the system and bath fields) is defined by

$$
\rho\left[\phi_{<}, \phi_{>}, \phi_{<}^{\prime}, \phi_{>}^{\prime}, t\right]=\left\langle\phi_{<} \phi_{>}|\hat{\rho}| \phi_{<}^{\prime} \phi_{>}^{\prime}\right\rangle,
$$


where $\left|\phi_{<}\right\rangle$and $\left|\phi_{>}\right\rangle$are the eigenstates of the field operators $\hat{\phi}_{<}$and $\hat{\phi}_{>}$, respectively. For simplicity, we will assume that the interaction is turned on at the initial time $t_{0}$ and that, at this time, the system and the environment are not correlated (we ignore, for the moment, the physical consequences of such a choice). Therefore, the total density matrix can be written as the product of the density matrix operator for the system and for the bath

$$
\hat{\rho}\left[t_{0}\right]=\hat{\rho}_{<}\left[t_{0}\right] \hat{\rho}_{>}\left[t_{0}\right]
$$

We will further assume that the initial state of the environment is the vacuum.

We are interested in the influence of the environment on the evolution of the system. Therefore the reduced density matrix is the object of relevance. It is defined by

$$
\rho_{\text {red }}\left[\phi_{<}, \phi_{<}^{\prime}, t\right]=\int \mathcal{D} \phi_{>} \rho\left[\phi_{<}, \phi_{>}, \phi_{<}^{\prime}, \phi_{>}, t\right]
$$

The reduced density matrix evolves in time by means of

$$
\rho_{r}\left[\phi_{<f}, \phi_{<f}^{\prime}, t\right]=\int d \phi_{<i} \int d \phi_{<i}^{\prime} J_{r}\left[\phi_{<f}, \phi_{<f}^{\prime}, t \mid \phi_{<i}, \phi_{<i}^{\prime}, t_{0}\right] \rho_{r}\left[\phi_{<i} \phi_{<i}^{\prime}, t_{0}\right],
$$

where $J_{r}\left[t, t_{0}\right]$ is the reduced evolution operator

$$
J_{r}\left[\phi_{<f}, \phi_{<f}^{\prime}, t \mid \phi_{<i}, \phi_{<i}^{\prime}, t_{0}\right]=\int_{\phi_{<i}}^{\phi_{<f}} \mathcal{D} \phi_{<} \int_{\phi_{<i}^{\prime}}^{\phi_{<f}^{\prime}} \mathcal{D} \phi_{<}^{\prime} \exp \frac{i}{\hbar}\left\{S\left[\phi_{<}\right]-S\left[\phi_{<}^{\prime}\right]\right\} F\left[\phi_{<}, \phi_{<}^{\prime}\right] .
$$

The influence functional (or Feynman-Vernon functional) $F\left[\phi_{<}, \phi_{<}^{\prime}\right]$ is defined as

$$
\begin{aligned}
F\left[\phi_{<}, \phi_{<}^{\prime}\right]= & \int d \phi_{>i} \int d \phi_{>i}^{\prime} \rho_{>}\left[\phi_{>i}, \phi_{>i}^{\prime}, t_{0}\right] \int d \phi_{>f} \int_{\phi_{>i}}^{\phi_{>f}} \mathcal{D} \phi_{>} \int_{\phi_{>i}^{\prime}}^{\phi_{>f}} \mathcal{D} \phi_{>}^{\prime} \\
& \times \exp \frac{i}{\hbar}\left\{S\left[\phi_{>}\right]+S_{i n t}\left[\phi_{<}, \phi_{>}\right]-S\left[\phi_{>}^{\prime}\right]-S_{i n t}\left[\phi_{<}^{\prime}, \phi_{>}^{\prime}\right]\right\}
\end{aligned}
$$

This functional takes into account the effect of the environment on the system.

We define the influence action $\delta A\left[\phi_{<}, \phi_{<}^{\prime}\right]$ and the coarse grained effective action (CGEA) $A\left[\phi_{<}, \phi_{<}^{\prime}\right]$ as

$$
\begin{gathered}
F\left[\phi_{<}, \phi_{<}^{\prime}\right]=\exp \frac{i}{\hbar} \delta A\left[\phi_{<}, \phi_{<}^{\prime}\right], \\
A\left[\phi_{<}, \phi_{<}^{\prime}\right]=S\left[\phi_{<}\right]-S\left[\phi_{<}^{\prime}\right]+\delta A\left[\phi_{<}, \phi_{<}^{\prime}\right] .
\end{gathered}
$$


We will calculate the influence action perturbatively in $\lambda$ and we will consider only terms up to order $\lambda^{2}$. The influence action has the following form

$$
\begin{aligned}
\delta A\left[\phi_{<}, \phi_{<}^{\prime}\right]= & \left\{\left\langle S_{i n t}\left[\phi_{<}, \phi_{>}\right]\right\rangle_{0}-\left\langle S_{i n t}\left[\phi_{<}^{\prime}, \phi_{>}^{\prime}\right]\right\rangle_{0}\right\} \\
& +\frac{i}{2}\left\{\left\langle S_{i n t}^{2}\left[\phi_{<}, \phi_{>}\right]\right\rangle_{0}-\left[\left\langle S_{i n t}\left[\phi_{<}, \phi_{>}\right]\right\rangle_{0}\right]^{2}\right\} \\
& -i\left\{\left\langle S_{\text {int }}\left[\phi_{<}, \phi_{>}\right] S_{i n t}\left[\phi_{<}^{\prime}, \phi_{>}^{\prime}\right]\right\rangle_{0}-\left\langle S_{i n t}\left[\phi_{<}, \phi_{>}\right]\right\rangle_{0}\left\langle S_{\text {int }}\left[\phi_{<}^{\prime}, \phi_{>}^{\prime}\right]\right\rangle_{0}\right\} \\
& \left.+\frac{i}{2}\left\{S_{i n t}^{2}\left[\phi_{<}^{\prime}, \phi_{>}^{\prime}\right]\right\rangle_{0}-\left[\left\langle S_{i n t}\left[\phi_{<}^{\prime}, \phi_{>}^{\prime}\right]\right\rangle_{0}\right]^{2}\right\},
\end{aligned}
$$

where the quantum average of a functional of the fields is defined as

$$
\begin{aligned}
\left\langle B\left[\phi_{>}, \phi_{>}^{\prime}\right]\right\rangle_{0}=\int d \phi_{>i} & \int d \phi_{>i}^{\prime} \rho_{>}\left[\phi_{>i}, \phi_{>i}^{\prime}, t_{0}\right] \int d \phi_{>f} \\
& \times \int_{\phi_{>i}}^{\phi_{>f}} \mathcal{D} \phi_{>} \int_{\phi_{>i}^{\prime}}^{\phi_{>f}} \mathcal{D} \phi_{>}^{\prime} \exp \frac{i}{\hbar}\left\{S_{0}\left[\phi_{>}\right]-S_{0}\left[\phi_{>}^{\prime}\right]\right\} B .
\end{aligned}
$$

We define the propagators of the environment field as

$$
\begin{gathered}
\left\langle\phi_{>}(x), \phi_{>}(y)\right\rangle_{0}=i G_{++}^{\Lambda}(x-y), \\
\left\langle\phi_{>}(x), \phi_{>}^{\prime}(y)\right\rangle_{0}=-i G_{+-}^{\Lambda}(x-y), \\
\left\langle\phi_{>}^{\prime}(x), \phi_{>}^{\prime}(y)\right\rangle_{0}=-i G_{--}^{\Lambda}(x-y) .
\end{gathered}
$$

These propagators are not the usual Feynman, positive-frequency Wightman, and Dyson propagators of the scalar field since, in this case, the momentum integration is restricted by the presence of the infrared cutoff $\Lambda$. The explicit expressions are

$$
\begin{gathered}
G_{++}^{\Lambda}(x-y)=\int_{|\vec{p}|>\Lambda} \frac{d^{4} p}{(2 \pi)^{4}} e^{i p(x-y)} \frac{1}{p^{2}-m^{2}+i \epsilon}, \\
G_{+-}^{\Lambda}(x-y)=\int_{|\vec{p}|>\Lambda} \frac{d^{4} p}{(2 \pi)^{4}} e^{i p(x-y)} 2 \pi i \delta(x-y) \Theta\left(p^{0}\right), \\
G_{--}^{\Lambda}(x-y)=\int_{|\vec{p}|>\Lambda} \frac{d^{4} p}{(2 \pi)^{4}} e^{i p(x-y)} \frac{1}{p^{2}-m^{2}-i \epsilon} .
\end{gathered}
$$


As an example, we show the expression for the propagator $G_{++}^{\Lambda}$ in the massless case. The usual Feynman propagator is

$$
G_{++}(x)=\frac{i}{8 \pi^{2}} \frac{1}{\sigma}-\frac{1}{8 \pi} \delta(\sigma),
$$

while

$$
\begin{aligned}
G_{++}^{\Lambda}(x) & =\frac{i}{8 \pi^{2}}\left[\frac{\cos [\Lambda(r-t)]}{r(r-t)}+\frac{\cos [\Lambda(r+t)]}{r(r+t)}\right]-\frac{1}{8 \pi^{2}}\left[\frac{\sin [\Lambda(r-t)]}{r(r-t)}-\frac{\sin [\Lambda(r+t)]}{r(r+t)}\right] \\
& \equiv G_{++}(x)-G_{++}^{|\vec{p}|<\Lambda}(x),
\end{aligned}
$$

where $\sigma=\frac{1}{2} x^{2}$ is one half of the geodesic distance.

The influence action can be computed from Eqs. (15)-(19) using standard techniques. After a long but straightforward calculation we find

$$
\begin{aligned}
\delta A\left[\phi_{<}, \phi_{<}^{\prime}\right]= & -\lambda \int d^{4} x\left[\frac{1}{24}\left(\phi_{<}^{4}(x)-\phi_{<}^{\prime 4}(x)\right)+\frac{1}{4} i G_{++}^{\Lambda}(0)\left(\phi_{<}^{2}(x)-\phi_{<}^{\prime 2}(x)\right)\right] \\
& +\lambda^{2} \int d^{4} x \int d^{4} y\left[-\frac{1}{72} \phi_{<}^{3}(x) G_{++}^{\Lambda}(x-y) \phi_{<}^{3}(y)-\frac{1}{36} \phi_{<}^{3}(x) G_{+-}^{\Lambda}(x-y) \phi_{<}^{\prime 3}(y)\right. \\
& +\frac{1}{72} \phi_{<}^{\prime 3}(x) G_{--}^{\Lambda}(x-y) \phi_{<}^{\prime 3}(y)-\frac{1}{16} \phi_{<}^{2}(x) i G_{++}^{\Lambda 2}(x-y) \phi_{<}^{2}(y) \\
& +\frac{1}{8} \phi_{<}^{2}(x) i G_{+-}^{\Lambda 2}(x-y) \phi_{<}^{\prime 2}(y)-\frac{1}{16} \phi_{<}^{\prime 2}(x) i G_{--}^{\Lambda 2}(x-y) \phi_{<}^{\prime 2}(y) \\
& +\frac{1}{18} \phi_{<}(x) G_{++}^{\Lambda 3}(x-y) \phi_{<}(y)+\frac{1}{9} \phi_{<}(x) G_{+-}^{\Lambda 3}(x-y) \phi_{<}^{\prime}(y) \\
& \left.-\frac{1}{18} \phi_{<}^{\prime}(x) G_{--}^{\Lambda 3}(x-y) \phi_{<}^{\prime}(y)\right] .
\end{aligned}
$$

Defining

$$
\begin{array}{rlrl}
P_{ \pm} & =\frac{1}{2}\left(\phi_{<}^{4} \pm \phi_{<}^{\prime 4}\right) \quad ; & R_{ \pm}=\frac{1}{2}\left(\phi_{<}^{3} \pm \phi_{<}^{\prime 3}\right) \\
Q_{ \pm}=\frac{1}{2}\left(\phi_{<}^{2} \pm \phi_{<}^{\prime 2}\right) \quad ; \quad \Phi_{ \pm}=\frac{1}{2}\left(\phi_{<} \pm \phi_{<}^{\prime}\right),
\end{array}
$$

and using simple identities for the propagators, the real and imaginary parts of the influence action can be written as

$$
\begin{aligned}
\operatorname{Re} \delta A= & -\lambda \int d^{4} x\left\{\frac{1}{12} P_{-}(x)+\frac{i}{2} G_{++}^{\Lambda}(0) Q_{-}(x)\right\} \\
& +\lambda^{2} \int d^{4} x \int d^{4} y\left\{-\frac{1}{18} R_{+}(x) \operatorname{Re} G_{++}^{\Lambda}(x-y) R_{-}(y)+\frac{1}{4} Q_{+}(x) \operatorname{Im} G_{++}^{\Lambda 2}(x-y) Q_{-}(y)\right. \\
& \left.+\frac{1}{3} \Phi_{+}(x) \operatorname{Re} G_{++}^{\Lambda 3}(x-y) \Phi_{-}(y)\right\}
\end{aligned}
$$




$$
\begin{aligned}
\operatorname{Im} \delta A= & \lambda^{2} \int d^{4} x \int d^{4} y\left\{-\frac{1}{18} R_{-}(x) \operatorname{Im} G_{++}^{\Lambda}(x-y) R_{-}(y)\right. \\
& \left.-\frac{1}{4} Q_{-}(x) \operatorname{Re} G_{++}^{\Lambda 2}(x-y) Q_{-}(y)+\frac{1}{3} \Phi_{-}(x) \operatorname{Im} G_{++}^{\Lambda 3}(x-y) \Phi_{-}(y)\right\}
\end{aligned}
$$

this expression is valid in general but, for simplicity, in what follows we will consider only the massless case.

The real part of the influence action in Eq. (26) contains divergences and must be renormalized. As the propagators Eqs. (17)-(19) differ from the usual ones only by the presence of the infrared cutoff, the ultraviolet divergences coincide with those of the usual $\lambda \phi^{4}$-theory. As a consequence, the influence action can be renormalized using the usual counterterms.

The term proportional to $G_{++}^{\Lambda}(0) Q_{-}(x)$ is divergent. As $G_{++}^{\Lambda}=G_{++}-G_{++}^{|\vec{p}|<\Lambda}$ and $G_{++}^{|\vec{p}|<\Lambda}(0)$ is finite, the divergences of $G_{++}^{\Lambda}$ and $G_{++}$coincide. This term renormalizes the mass.

Consider now the square of the Feynman propagator. Using dimensional regularization we find

$$
G_{++}^{\Lambda 2}(x)=G_{++}^{2}(x)+G_{++}^{(|\vec{p}|<\Lambda) 2}(x)-2 G_{++}(x) G_{++}^{(|\vec{p}|<\Lambda)}(x)
$$

where

$$
\begin{gathered}
G_{++}^{2}(x)=\frac{i}{16 \pi^{2}}\left[\frac{1}{n-4}+\psi(1)-4 \pi\right] \delta^{4}(x)+i \Sigma(x)-\eta(x)-\log \left[4 \pi \mu^{2}\right] \\
\Sigma(x)=\frac{1}{(2 \pi)^{4}} \int d^{4} p e^{i p x} \log \left|p^{2}\right| \\
\eta(x)=\frac{\pi}{(2 \pi)^{4}} \int d^{4} p e^{i p x} \Theta\left(p^{2}\right) .
\end{gathered}
$$

Note that the divergence is the usual one, i.e., proportional to $\frac{1}{n-4} \delta^{4}(x-y)$ and independent of $\Lambda$. Consequently, the term $\operatorname{Im} G_{++}^{\Lambda 2}(x-y) Q_{+}(x) Q_{-}(y)$ in Eq. (26) is also divergent and renormalizes the coupling constant $\lambda$. The other divergences can be treated in a similar way. One can also check that the imaginary part of the effective action does not contain divergences. 
The real and imaginary parts of $\delta A\left[\phi_{<}, \phi_{<}^{\prime}\right]$ can be associated with the dissipation and noise respectively, and can be related by some integral equation known as the fluctuationdissipation relation.

One can regard the imaginary part of $\delta A$ as coming from three noise sources $\nu(x), \xi(x)$, and $\eta(x)$ with a gaussian functional probability distribution given by

$$
\begin{aligned}
P[\nu(x), \xi(x), \eta(x)]= & N_{\nu} N_{\xi} N_{\eta} \exp \left\{-\frac{1}{2} \int d^{4} x \int d^{4} y \nu(x)\left[\frac{\lambda^{2}}{9} \operatorname{Im} G_{++}^{\Lambda}\right]^{-1} \nu(y)\right\} \\
& \left.\times \exp \left\{-\frac{1}{2} \int d^{4} x \int d^{4} y \xi(x)\left[\frac{\lambda^{2}}{2} \operatorname{Re} G_{++}^{\Lambda 2}\right]^{-1}\right] \xi(y)\right\} \\
& \times \exp \left\{-\frac{1}{2} \eta(x)\left[\frac{-2 \lambda^{2}}{3} \operatorname{Im} G_{++}^{\Lambda 3}\right]^{-1} \eta(y)\right\},
\end{aligned}
$$

where $N_{\nu}, N_{\xi}$, and $N_{\eta}$ are normalization factors. Indeed, we can write the imaginary part of the influence action as three functional integrals over the gaussian fields $\nu(x), \xi(x)$, and $\eta(x)$

$$
\begin{aligned}
\int \mathcal{D} \nu(x) \int \mathcal{D} & \xi(x) \int \mathcal{D} \eta(x) P[\nu, \xi, \eta] \exp -\frac{i}{\hbar}\left\{R_{-}(x) \nu(x)+Q_{-}(x) \xi(x)+\Phi_{-}(x) \eta(x)\right\} \\
= & \exp \left\{-\frac{i}{\hbar} \int d^{4} x \int d^{4} y\left[\frac{\lambda^{2}}{18} R_{-}(x) \operatorname{Im} G_{++}^{\Lambda}(x, y) R_{-}(y)\right.\right. \\
+ & \left.\left.\frac{\lambda^{2}}{4} Q_{-}(x) \operatorname{Re} G_{++}^{\Lambda 2}(x, y) Q_{-}(y)-\frac{\lambda^{2}}{3} \Phi_{-}(x) \operatorname{Im} G_{++}^{\Lambda 3}(x, y) \Phi_{-}(y)\right]\right\} .
\end{aligned}
$$

Therefore, the CGEA can be rewritten as

$$
A\left[\phi_{<}, \phi_{<}^{\prime}\right]=-\frac{1}{i} \ln \int \mathcal{D} \nu P[\nu] \int \mathcal{D} \xi P[\xi] \int \mathcal{D} \eta P[\eta] \exp \left\{i S_{e f f}\left[\phi_{<}, \phi_{<}^{\prime}, \nu, \xi, \eta\right]\right\},
$$

where

$$
S_{e f f}\left[\phi_{<}, \phi_{<}^{\prime}, \nu, \xi, \eta\right]=\operatorname{Re} A\left[\phi_{<}, \phi_{<}^{\prime}\right]-\int d^{4} x\left[R_{-}(x) \nu(x)+Q_{-}(x) \xi(x)+\Phi_{-}(x) \eta(x)\right] .
$$

In the associated functional Langevin equation for the field [8,9], the corresponding stochastic force is

$$
F_{\text {noise }} \sim \nu(x) \phi_{<}^{2}(x)+\xi(x) \phi_{<}(x)+\eta(x),
$$

i.e., multiplicative and additive noise.

To summarize this Section, we have seen that the environment has three fundamental effects over the system [16]: renormalization, dissipation, and noise. 


\section{THE EVOLUTION OF THE REDUCED DENSITY MATRIX: MASTER EQUATION}

In this Section we will obtain the evolution equation for the reduced density matrix (master equation). Before doing this, let us briefly review the case of the QBM. Denote by $x$ the coordinate of the Brownian particle, by $\Omega$ its frequency, and by $q_{i}$ the coordinates of the oscillators in the environment. For a linear coupling $x q_{i}$, the master equation for the reduced density matrix $\rho_{r}\left(x, x^{\prime}, t\right)$ is of the form [17]

$$
\begin{aligned}
i \hbar \rho_{r}\left(x, x^{\prime}, t\right) & =\left\langle x\left|\left[H, \rho_{r}\right]\right| x^{\prime}\right\rangle-i \gamma(t)\left(\partial_{x}-\partial_{x^{\prime}}\right) \rho_{r}\left(x, x^{\prime}, t\right) \\
& -i D(t)\left(x-x^{\prime}\right)^{2} \rho_{r}\left(x, x^{\prime}, t\right)+f(t)\left(x-x^{\prime}\right)\left(\partial_{x}+\partial_{x^{\prime}}\right) \rho_{r}\left(x, x^{\prime}, t\right),
\end{aligned}
$$

where the coefficients $\gamma(t), D(t)$ and $f(t)$ depend on the properties of the environment (temperature $\beta^{-1}$ and spectral density $\left.I(\omega)\right)$. The difussion coefficient $D(t)$ is

$$
D(t)=\int_{0}^{t} d s \cos (\Omega s) \quad \int_{0}^{\infty} d \omega I(\omega) \operatorname{coth}\left(\frac{1}{2} \beta \hbar \omega\right) \cos (\omega s)
$$

and gives the main contribution to the decoherence. Indeed, an approximate solution of Eq. (37) is

$$
\rho_{r}\left[x, x^{\prime} ; t\right] \approx \rho_{r}\left[x, x^{\prime}, 0\right] \exp \left[-\left(x-x^{\prime}\right)^{2} \int_{0}^{t} D(s) d s\right]
$$

and we see that the off-diagonal terms of the density matrix are suppressed as long as $\int_{0}^{t} D(s) d s$ is large enough.

For non-linear couplings like $x^{n} q_{i}^{m}$, one expects the master equation to contain terms of the form $i D^{(n, m)}(t)\left(x^{n}-x^{\prime n}\right)^{2} \rho_{r}$.

Here we will generalize these results to the field theory of Section 2. To this end, we will compute the reduced evolution operator Eq. (11). As the operator is defined through a path integral, we can obtain an estimation using the saddle point approximation:

$$
J_{r}\left[\phi_{<f}, \phi_{<f}^{\prime}, t \mid \phi_{<i}, \phi_{<i}^{\prime}, t_{0}\right] \approx \exp \frac{i}{\hbar} A\left[\phi_{<}^{c l}, \phi_{<}^{\prime c l}\right],
$$


where $\phi_{<}^{c l}\left(\phi_{<}^{\prime} c l\right)$ is the solution of the equation of motion $\left.\frac{\delta R e A}{\delta \phi_{<}}\right|_{\phi_{<}=\phi_{<}^{\prime}}=0$ with boundary conditions $\phi_{<}^{c l}\left(t_{0}\right)=\phi_{<i}\left(\phi_{<i}^{\prime}\right)$ and $\phi_{<}^{c l}(t)=\phi_{<f}\left(\phi_{<f}^{\prime}\right)$.

The decoherence effects are contained in the imaginary part of the CGEA, which is already of order $\lambda^{2}$. As a consequence, in the evaluation of $\operatorname{Im} A$ we can approximate $\phi_{<}^{c l}$ by the solution of the free field equation satisfying the appropiate boundary conditions. Therefore, the classical solution reads

$$
\phi_{<}^{c l}(\vec{x}, s)=\left[\phi_{<f} \frac{\sin \left(k_{0} s\right)}{\sin \left(k_{0} t\right)}+\phi_{<i} \frac{\sin \left[k_{0}(t-s)\right]}{\sin \left(k_{0} t\right)}\right] \cos \left(\vec{k}_{0} \cdot \vec{x}\right) \equiv \phi_{<}^{c l}(s) \cos \left(\vec{k}_{0} \cdot \vec{x}\right),
$$

where we assumed that the system-field contains only one Fourier mode with $\vec{k}=\vec{k}_{0}$. This is a sort of "minisuperspace" approximation for the system-field that will greatly simplify the calculations.

Following the same techniques used for the QBM [17], to obtain the master equation we must compute the time derivative of the propagator $J_{r}$, and eliminate the dependence on the initial field configurations $\phi_{<i}$ and $\phi_{<i}^{\prime}$ that enters through $\phi_{<}^{c l}$ and $\phi_{<}^{\prime c l}$. This can be easily done because the free propagator, defined as

$$
J_{0}\left[\phi_{<f}, \phi_{<f}^{\prime}, t \mid \phi_{<i}, \phi_{<i}^{\prime}, 0\right]=\int_{\phi_{<i}}^{\phi_{<f}} \mathcal{D} \phi_{<} \int_{\phi_{<i}^{\prime}}^{\phi_{<f}^{\prime}} \mathcal{D} \phi_{<}^{\prime} \exp \left\{\frac{i}{\hbar}\left[S_{0}\left(\phi_{<}\right)-S_{0}\left(\phi_{<}^{\prime}\right)\right]\right\}
$$

satisfies the identities

$$
\phi_{<}^{c l}(\vec{x}, s) J_{0}=\left[\phi_{<f} \cos \left[k_{0}(t-s)\right]+\frac{\sin \left[k_{0}(t-s)\right]}{k_{0}} i \partial_{\phi_{<f}}\right] J_{0}
$$

and

$$
\phi_{<}^{\prime c l}(\vec{x}, s) J_{0}=\left[\phi_{<f}^{\prime} \cos \left[k_{0}(t-s)\right]-\frac{\sin \left[k_{0}(t-s)\right]}{k_{0}} i \partial_{\phi_{<f}^{\prime}}\right] J_{0}
$$

The temporal derivative is given by

$$
\begin{aligned}
i \hbar \partial_{t} J_{r}[ & \left.\phi_{<f}, \phi_{<f}^{\prime}, t \mid \phi_{<i}, \phi_{<i}^{\prime}, 0\right]=\left\{h_{r e n}\left[\phi_{<}\right]-h_{r e n}\left[\phi_{<}^{\prime}\right]-i \lambda^{2}\left[\frac{\left(\phi_{<f}^{3}-\phi_{<f}^{\prime 3}\right) V}{1152}\right.\right. \\
& \times \int_{0}^{t} d s R_{-}^{c l}(s) I m G_{++}^{\Lambda}\left(3 k_{0} ; t-s\right) \\
& +\frac{\left(\phi_{<f}^{2}-\phi_{<f}^{\prime 2}\right) V}{32} \int_{0}^{t} d s Q_{-}^{c l}(s)\left(\operatorname{Re} G_{++}^{\Lambda 2}\left(2 k_{0} ; t-s\right)+2 \operatorname{Re} G_{++}^{\Lambda 2}(0 ; t-s)\right)
\end{aligned}
$$




$$
\begin{aligned}
& \left.-\frac{\left(\phi_{<f}-\phi_{<f}^{\prime}\right) V}{6} \int_{0}^{t} d s \Phi_{-}^{c l}(s) \operatorname{Im} G_{++}^{\Lambda 3}\left(k_{0} ; t-s\right)\right] \\
& -\lambda^{2}\left[-\frac{\left(\phi_{<f}^{3}+\phi_{<f}^{\prime 3}\right) V}{1152} \int_{0}^{t} d s R_{-}^{c l}(s) \operatorname{Re} G_{++}^{\Lambda}\left(3 k_{0} ; t-s\right)\right. \\
& +\frac{\left(\phi_{<f}^{2}+\phi_{<f}^{\prime 2}\right) V}{32} \int_{0}^{t} d s Q_{-}^{c l}(s)\left(\operatorname{Im} G_{++}^{\Lambda 2}\left(2 k_{0} ; t-s\right)+2 \operatorname{Im} G_{++}^{\Lambda 2}(0 ; t-s)\right) \\
& \left.\left.+\frac{\left(\phi_{<f}-\phi_{<f}^{\prime}\right) V}{6} \int_{0}^{t} d s \Phi_{-}^{c l}(s) \operatorname{Re} G_{++}^{\Lambda 3}\left(k_{0} ; t-s\right)\right]+\ldots\right\} J_{r}\left[\phi_{<f}, \phi_{<f}^{\prime}, t \mid \phi_{<i}, \phi_{<i}^{\prime}, 0\right],
\end{aligned}
$$

where $G_{++}^{A n}(k ; t-s)$ is the Fourier transform of the $n$-power of the propagator $(n=1,2$, or 3). The ellipsis denotes other terms coming from the time derivative that do not contribute to the diffusive effects (we will ignore all terms not proportional to $\left(\phi_{<f}^{n}-\phi_{<f}^{\prime n}\right)^{2}$ in the final equation). The volume factor $V$ that appears in Eq. (45) is due to our assumption that the system-field contains only one mode. As usual in quantum field theory, it disappears when considering properly defined wave packets.

Using the identities Eqs. (43) and (44) we can remove the dependence on the initial boundary conditions and obtain the master equation. As the final equation is complicated and not very illuminating, we only show the correction to the usual unitary evolution term coming from the noise kernels:

$$
\begin{aligned}
& i \hbar \partial_{t} \rho_{r}\left[\phi_{<f}, \phi_{<f}^{\prime}, t\right]=\left\langle\phi_{<f}\left|\left[\hat{H}_{r e n}, \hat{\rho}_{r}\right]\right| \phi_{<f}^{\prime}\right\rangle-i \lambda^{2}\left[\frac{\left(\phi_{<f}^{3}-\phi_{<f}^{3}\right)^{2} V}{1152} D_{1}\left(k_{0} ; t\right)\right. \\
& \left.\quad+\frac{\left(\phi_{<f}^{2}-\phi_{<f}^{\prime 2}\right)^{2} V}{32} D_{2}\left(k_{0} ; t\right)-\frac{\left(\phi_{<f}-\phi_{<f}^{\prime}\right)^{2} V}{6} D_{3}\left(k_{0} ; t\right)\right] \rho_{r}\left[\phi_{<f}, \phi_{<f}^{\prime}, t\right]+\ldots .
\end{aligned}
$$

This equation contains three time-dependent diffusion coefficients $D_{i}(t)$. Up to one loop, only $D_{1}$ and $D_{2}$ survive and are given by

$$
\begin{aligned}
D_{1}\left(k_{0} ; t\right)= & \int_{0}^{t} d s \cos ^{3}\left(k_{0} s\right) \operatorname{Im} G_{++}^{\Lambda}\left(3 k_{0} ; t-s\right) \\
& =\frac{1}{6 k_{0}} \int_{0}^{t} d s \cos ^{3}\left(k_{0} s\right) \cos \left(3 k_{0} s\right) \theta\left(3 k_{0}-\Lambda\right) \\
& =\frac{2 k_{0} t+3 \sin \left(2 k_{0} t\right)+\frac{3}{2} \sin \left(4 k_{0} t\right)+\frac{1}{3} \sin \left(6 k_{0} t\right)}{576 k_{0}^{2}}, \quad \frac{\Lambda}{3}<k_{0}<\Lambda \\
D_{2}\left(k_{0} ; t\right) & =\int_{0}^{t} d s \cos ^{2}\left(k_{0} s\right)\left(\operatorname{Re} G_{++}^{\Lambda 2}\left(2 k_{0} ; t-s\right)+2 \operatorname{Re} G_{++}^{\Lambda 2}(0 ; t-s)\right) .
\end{aligned}
$$

Using that 


$$
\begin{aligned}
R e G_{++}^{\Lambda 2}\left(2 k_{0} ; t-s\right)=\frac{\pi}{k_{0}}\{ & \int_{\Lambda}^{2 k_{0}+\Lambda} d p \int_{\Lambda}^{2 k_{0}+p} d z \cos [(p+z) s] \\
& \left.+\int_{2 k_{0}+\Lambda}^{\infty} d p \int_{p-2 k_{0}}^{p+2 k_{0}} d z \cos [(p+z) s]\right\} \\
R e G_{++}^{\Lambda 2}(0 ; t-s) & =\pi\left\{2 \pi \delta(s)-2 \frac{\sin (2 \Lambda s)}{s}\right\}
\end{aligned}
$$

the $D_{2}$ diffusion coefficient reads

$$
\begin{aligned}
D_{2}\left(k_{0} ; t\right)=\frac{\pi}{4}\{ & 3 \pi-\left(\frac{3}{2}-\frac{\Lambda}{2 k_{0}}\right) \operatorname{Si}\left[2 t\left(\Lambda-k_{0}\right)\right] \\
& -\left(2-\frac{\Lambda}{2 k_{0}}\right) \operatorname{Si}[2 \Lambda t]-\left(\frac{3}{2}+\frac{\Lambda}{2 k_{0}}\right) \operatorname{Si}\left[2 t\left(\Lambda+k_{0}\right)\right]-\left(1+\frac{\Lambda}{2 k_{0}}\right) \operatorname{Si}\left[2 t\left(2 k_{0}+\Lambda\right)\right] \\
& +\frac{\cos [2 \Lambda t]}{4 k_{0} t}-\frac{\cos \left[2 t\left(\Lambda+k_{0}\right)\right]}{4 k_{0} t}+\frac{\cos \left[2 t\left(\Lambda-k_{0}\right)\right]}{4 k_{0} t}-\frac{\cos \left[2 t\left(2 k_{0}+\Lambda\right)\right]}{4 k_{0} t},
\end{aligned}
$$

where $S i[z]$ denotes the sine-integral function [18].

Eq. (46) is the generalization of the QBM master equation we were looking for. In our case, the system is coupled in a nonlinear form. Therefore, owing to the existence of three interaction terms $\left(\phi_{<}^{3} \phi_{>}, \phi_{<}^{2} \phi_{>}^{2}\right.$, and $\left.\phi_{<} \phi_{>}^{3}\right)$ there are three diffusion coefficients in the master equation. The form of the coefficients is fixed by these couplings and by the particular choice of the quantum state of the environment.

Our results are valid in the single-mode approximation of Eq. (41). In this approximation

one obtains a reduced density matrix for each mode $\vec{k}_{0}$, and neglects the interaction between different system-modes. Due to this interaction, in the general case, $\rho_{r}$ will be different from $\prod_{\vec{k}_{0}} \rho_{r}\left(\vec{k}_{0}\right)$. This point deserves further study.

\section{DECOHERENCE AND COSMOLOGICAL PERTURBATIONS}

In this Section we will analyze the behavior of the diffusion coefficients $D_{1}$ and $D_{2}$. We will also extend the results to DeSitter space, in order to discuss the issue of the quantum to classical transition of the primordial quantum fluctuations.

A detailed analysis of the quantum to classical transition in the model we are considering is a very complicated task. One should analyze in detail the master equation and see whether 
the off-diagonal elements of the reduced density matrix are suppressed or not. One should also study the form of the Wigner function, and see whether it predicts classical correlations or not. This is beyond the scope of this paper. Having in mind the analogy with the QBM, here we will only be concerned with the diffusive terms of the master equation. We will use the value of the diffusion coefficients as the signal for decoherence, which is, of course, only a rough approximation.

After these words of caution, let us turn to the analysis of Eqns. (47) and (51). The coefficient $D_{1}$ is associated to the interaction term $\phi_{<}^{3} \phi_{>}$. We are considering only one Fourier mode for the system, with wavevector $\vec{k}_{0}$. The environment field enters linearly in the interaction term, and contains only modes with $k>\Lambda$ (by definition). Consequently, $\phi_{<}$is only coupled with the $\vec{k}=3 \vec{k}_{0}$ mode of the environment. This implies that $D_{1}$ is different from zero only if $\frac{\Lambda}{3}<k_{0}<\Lambda$. In Fig. 1 we plot the temporal evolution of $D_{1}\left(k_{0}, t\right)$. The diffusive effects increase with time as $\frac{t}{k_{0}}$ (of course our perturbative calculations are not valid at large times). The shape of the curve depends on $k_{0}$ and takes its maximum value for $k_{0}=\frac{\Lambda}{3}$. To illustrate this, in Fig. 2 we plot $D_{1}\left(k_{0}, t\right)$ as a function of $k_{0}$ for a fixed time.

It is interesting to note that we would obtain a similar diffussion coefficient in a particular QBM, with interaction $x^{3} q$ and spectral density $I(\omega) \sim \delta\left(\omega-3 k_{0}\right) \theta(\omega-\Lambda)$.

Let us now consider the coefficient $D_{2}$ coming from the interaction term $\phi_{<}^{2} \phi_{>}^{2}$. As the interaction is now quadratic in $\phi_{>}$, there are no restrictions on the values of $k_{0}$ such that $D_{2} \neq 0$. In Fig. 3 we have plotted the temporal evolution of this coefficient for several values of $y=\frac{k_{0}}{\Lambda}$ (the variable $y$ labels the different curves). Although formally the diffusion coefficients should vanish at $t=0$ (see Eq. (48)), the graph shows an initial jolt for all values of $y$. The origin of this behavior is the uncorrelated initial condition Eq.(8). We have assumed that the interaction between the system and the environment is turned on instantaneously at $t=0$. "Instantaneously" means in a time scale shorter than all time scales present in the system and environment. Therefore, this initial condition assumes implicitly the existence of an ultraviolet cutoff for the frequencies in the environment. Had we considered such cutoff $\Lambda_{u v}, D_{2}$ would have vanished at $t=0$ and developed a peak at 
$t \sim \frac{1}{\Lambda_{u v}}$. This is exactly what happens in the QBM [6]. In order to avoid this cutoff, one should consider a situation where the interaction is turned on adiabatically.

Fig. 3 shows that, after the (probably unphysical) jolt, the diffusive effects grow with $y$, and are maximum for $k_{0} \simeq \Lambda$. The physical interpretation is that the decoherence increases when the frequency of the system is large enough to excite the environment.

Figs. 4 and 5 show the dependence of $D_{2}$ with $k_{0}$, at a fixed time. For large values of $l=\Lambda t(\Lambda \rightarrow \infty)$, the environment contains only very high frequencies and the diffusion coefficient is very small unless $k_{0} \sim \Lambda$ (Fig. 4). When $l \leq 1, D_{2}$ is appreciably different from zero for all modes inside the system, i.e., the environment is able to produce decoherence for $0<k_{0}<\Lambda$ (Fig. 5). Finally, for $l \ll 1 D_{2}\left(k_{0}, t\right)$ approaches a non vanishing value in the limit $k_{0}<\Lambda \rightarrow 0$.

It is instructive to compare our results with those of Ref. [13], where a similar analysis is done for two independent fields $\phi$ and $\varphi$ coupled through a biquadratic interaction $\varphi^{2} \phi^{2}$. In that case, one can show that the diffusive effects are more important in the infrared. In our case, the analysis of the coefficients $D_{1}$ and $D_{2}$ shows that the loss of coherence is more important for $k_{0} \sim \Lambda$ rather than in the infrared.

After this long journey, we now turn to the issue of cosmological perturbations. The results of Section 3 can be easily generalized to the case of a conformally coupled scalar field in a Robertson Walker spacetime. The expressions for the diffusion coefficients $D_{1}$ and $D_{2}$ are the same as those for Minkowski space Eqns. (47) and (51), with the replacement $t \rightarrow \eta=\int_{0}^{t} \frac{d t^{\prime}}{a\left(t^{\prime}\right)}$. Here $a(t)$ is the scale factor of the Robertson Walker metric and $\eta$ is the conformal time. For the particular case of a DeSitter spacetime we have $a(t)=\exp (H t)$ and $\eta=\frac{1}{H}\left(1-\frac{1}{a}\right)$. Therefore, the dimensionless quantities $\Lambda t$ and $k_{0} t$ in Eqs. (47) and (51) become $\frac{\Lambda}{H}\left(1-\frac{1}{a}\right)$ and $\frac{k_{0}}{H}\left(1-\frac{1}{a}\right)$ respectively. As $a(t=0)=1, \Lambda$ and $k_{0}$ are the physical values of the cutoff and wavevector at the initial time.

Now we can ask the following question: which is the maximum value of the cutoff $\Lambda$ such that, a few e-foldings after the initial time, all modes with $k_{0} \leq \Lambda$ still suffer the diffusive effects? The analysis of the diffusion coefficients in Minkowski spacetime shows that $D_{1}$ is 
very small for $l \gg 1$ (Fig. 2). A similar thing happens for $D_{2}$ : if $l \gg 1$ this coefficient is very small in the infrared sector, and the diffusive effects are relevant only for $k_{0} \sim \Lambda$ (Fig. 4). As in DeSitter spacetime we have $l \approx \frac{\Lambda}{H}$, we conclude that we can include in our system only those modes with wavelength larger than the horizon $H^{-1}$. This is Starobinsky's original suggestion. If we include wavelengths shorter than the horizon, the low frequency sector of the system cannot excite the environment and does not decohere.

Our results do not apply directly to the inflationary models, since our scalar field is conformally coupled to the curvature. A more realistic model should contain a minimally coupled scalar field. Moreover, the cutoff should be time dependent [19] since the system should contain, at each time, the modes with $k_{0 p h}=\frac{k_{0}}{a}<H$. In spite of this, we think that this example illustrates the main aspects of the problem.

\section{FINAL REMARKS}

Let us summarize the new results contained in this paper. After the integration of the high frequency modes in Section 2, we obtained the CGEA for the low energy modes. From the imaginary part of the CGEA we obtained, in Section 3, the diffusion coefficients of the master equation. Similar calculations have been done previously 13 considering two scalar fields as system and environment. Here the system and environment are two sectors of a single scalar field, and the results depend on the "size" of these sectors, which is fixed by the critical wavelength $\Lambda^{-1}$.

In Section 4 we analyzed the $\Lambda$-dependence of the diffusion coefficients. We showed that the decoherence is larger for those modes in the system whose wavelength is close to the critical value. We also generalized the results to DeSitter spacetime and showed that, when the critical wavelength is equal to the horizon size, all modes in the system suffer decoherence and may become classical.

To end this paper, we would like to point out an interesting connection between our work, the renormalization group and the block-spin transformation. The CGEA of Section 2 is 
similar to the "average effective action" (AEA) proposed in Ref. [20] (see also Refs [21,22]). The AEA is an effective action for averages of the field over a finite space-time volume in Euclidean space. It is defined through a splitting similar to the one used in this paper, but now with a critical Euclidean four-momentum $p_{\mu} p_{\mu}=\Lambda^{2}$, where $\Lambda^{-1}$ is the typical size of the Euclidean average volume. The AEA interpolates between the classical action $(\Lambda \rightarrow 0)$ and the usual effective action $(\Lambda \rightarrow \infty)$, and realizes the block- spin transformation [23] in the continuum limit.

It is possible to write an exact evolution equation for the dependence of the AEA with the scale $\Lambda$. This equation was originally discussed by Wegner and Houghton [24, and by Polchinsky 25] in his proof of renormalizability of $\lambda \phi^{4}$ - theory. It can be used as the starting point for non perturbative calculations in quantum field theory.

The CGEA we found in this paper can be seen as the Close Time Path version (as opposed to Euclidean version) of the AEA, where the field averages are taken over 3-volumes contained in $t=$ const surfaces. Here we evaluated the CGEA perturbatively. However, it is possible to write an exact equation for the dependence of the CGEA on the critical wavelength. This evolution equation could be the starting point for non perturbative evaluations of the influence functional. Work in this direction is in progress [26].

\section{ACKNOWLEDGMENTS}

We are greatly indebted to J.P. Paz for many useful discussions. We have also benefited from conversations with E. Calzetta, B. L. Hu, L. Oxman, R. Scoccimarro, and M. Zaldarriaga. This research was supported by Universidad de Buenos Aires, Consejo Nacional de Investigaciones Científicas y Técnicas and by Fundación Antorchas. 


\section{REFERENCES}

1 W. H. Zurek, Phys. Rev. D24, 1516 (1981); D26, 1862 (1982); Physics Today 44, 36 (1991)

$2 \quad$ W. G. Unruh and W. H. Zurek, Phys. Rev. D40, 1071 (1989)

3 R. Laflamme and J. Louko, Phys. Rev. D43, 3317 (1991); M. Berry, Philos. Trans. R. Soc. 287, 237 (1977)

$4 \quad$ J. P. Paz and S. Sinha, Phys. Rev. D44, 1038 (1991)

5 C. Kiefer, Class. Quantum Grav. 4, 1369 (1987); T. Padmanabhan, Phys. Rev. D39, 2912 (1989); J. Halliwell, Phus. Rev. D36, 3627 (1987); E. Calzetta and F. D. Mazzitelli, Phys. Rev. D42, 4066 (1990)

$6 \quad$ B. L. Hu, J. P. Paz, and Y. Zhang, Phys. Rev. D45, 2843 (1993); Phys. Rev. D47, 1576 (1993)

7 E. Calzetta and B. L. Hu, Phys. Rev. D49, 6636 (1994); B. L. Hu and A. Matacz, Phys. Rev. D49, 6612 (1994)

$8 \quad$ M. Morikawa, Phys. Rev. D33, 3607 (1986)

$9 \quad$ M. Gleiser and R. Ramos, Phys. Rev. D50, 2441 (1994)

10 E. Kolb and M. Turner, the Early universe, Addison-Wesley Publishing Company (1990); R. Brandenberguer, Lectures on Modern Cosmology and Structure Formation, proceedings of the 7th Swieca Summer School in Particles and Fields, Campos do Jordao, Brazil; eds. O. Eboli and V. Ribelles (World Scientific, Singapure, 1993)

11 R. Brandenberguer, R. Laflamme, and M. Mijić, Int. J. Mod. Phys. A5, 2311 (1990)

12 H. A. Feldman and A. Y. Kamenshchick, Class. Quantum Grav. 8, 265 (1991)

13 B. L. Hu, J. P. Paz, and Y. Zhang, Quantum Origin of Noise and Fluctuations in 
Cosmology, in The Origin of Structure in the Universe, eds. E. Gunzig and P. Nardone (Kluwer, Dordrecht, 1993)

14 A. A. Starobinsky, in Field Theory, Quantum Gravity and Strings, ed. H. J. de Vega and N. Sanchez (Springer, Belin 1986)

15 R. Feynman and F. Vernon, Ann. Phys. (N. Y.) 24, 118 (1963)

16 H. F. Dowker and J. Halliwell, Phys. Rev. D46, 1580 (1992)

17 J. P. Paz, in The Physical Origin of Time Asymmetry, ed. by J. Halliwell, J. Perez Mercader and W. Zurek (Cambridge University Press, Cambridge, 1994)

18 M. Abramowitz and I. Stegun (eds.) of Handbook of Mathematical Functions, Dover Publications (N. Y.) (1972)

19 S. Habib, Phys. Rev. D46, 2408 (1992)

20 C. Wetterich, Nucl. Phys. B352, 529 (1991)

21 T. R. Morris, Int. J. Mod. Phys. A (1994)

22 S. B. Liao and J. Polonyi, Ann. Phys. 222, 122 (1993)

23 K. G. Wilson and I. G. Kogut, Phys. Rep. 12, 75 (1974)

24 F. J. Wegner and A. Houghton, Phys. Rev. A8, 401 (1973)

25 J. Polchinsky, Nucl. Phys. B231, 269 (1984)

26 D. Dalvit, F. Lombardo, and F. D. Mazzitelli, (unpublished) 


\section{FIGURE CAPTIONS}

FIG.1. Temporal evolution of the coefficient $D_{1}$ for a fixed $\frac{\Lambda}{3}<k_{0}<\Lambda$

FIG.2. The coefficient $D_{1}$ as a function of $k_{0}$ for a fixed value of $t . D_{1}$ is different from

zero only inside the window $\frac{\Lambda}{3}<k_{0}<\Lambda$. In the figure we indicated this window for the particular value $l=\Lambda t=15$

FIG.3. Temporal evolution of the coefficient $D_{2}$ for different values of $y=\frac{k_{0}}{\Lambda}$

FIG.4. The coefficient $D_{2}$ as a function of $k_{0}$ for a fixed value of $t$ and $l=\Lambda t=100$

FIG.5. The same as Fig. 4 but with smaller values for $l$ 
Fig.1

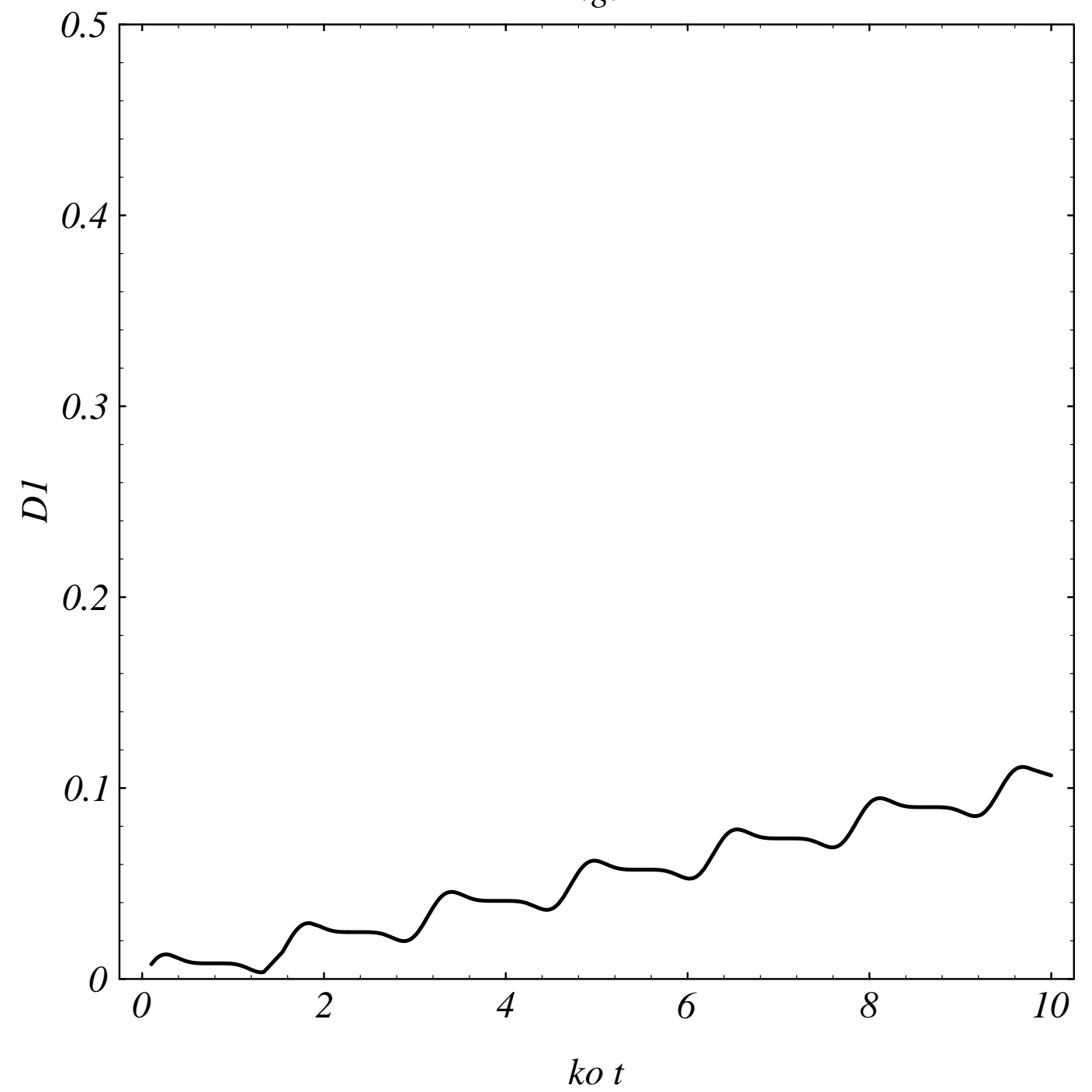


Fig. 2

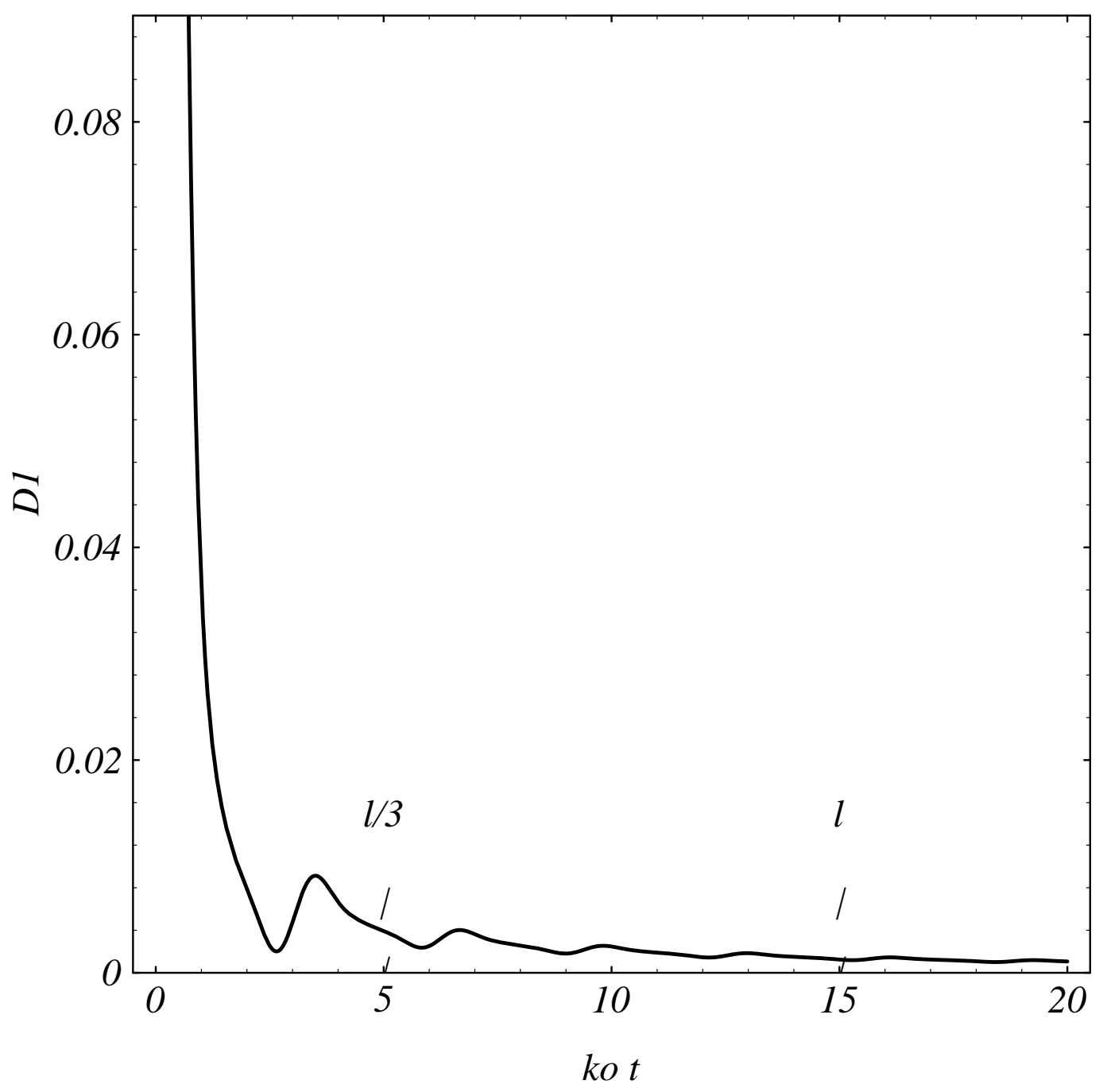


Fig. 3

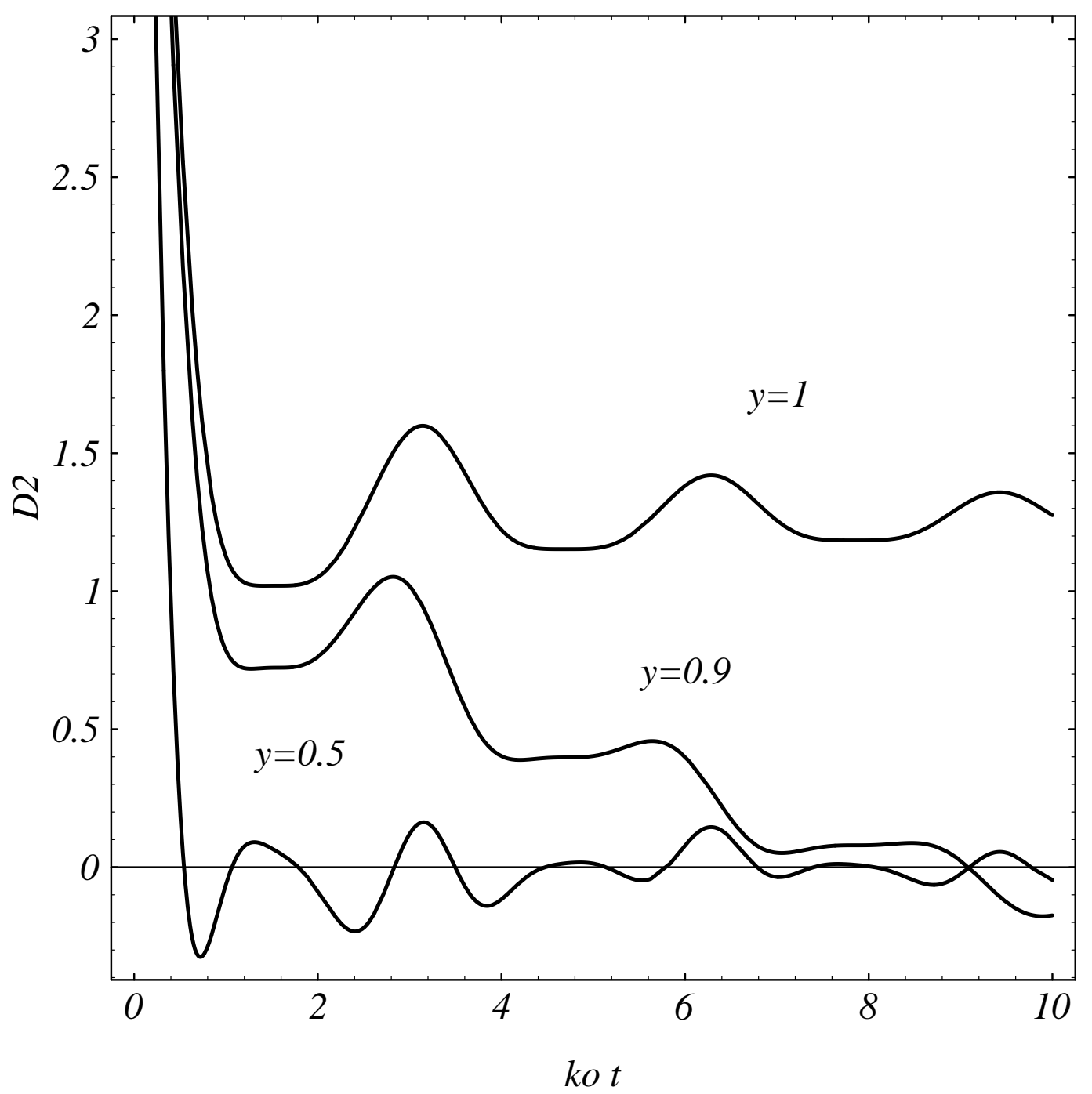


Fig.4

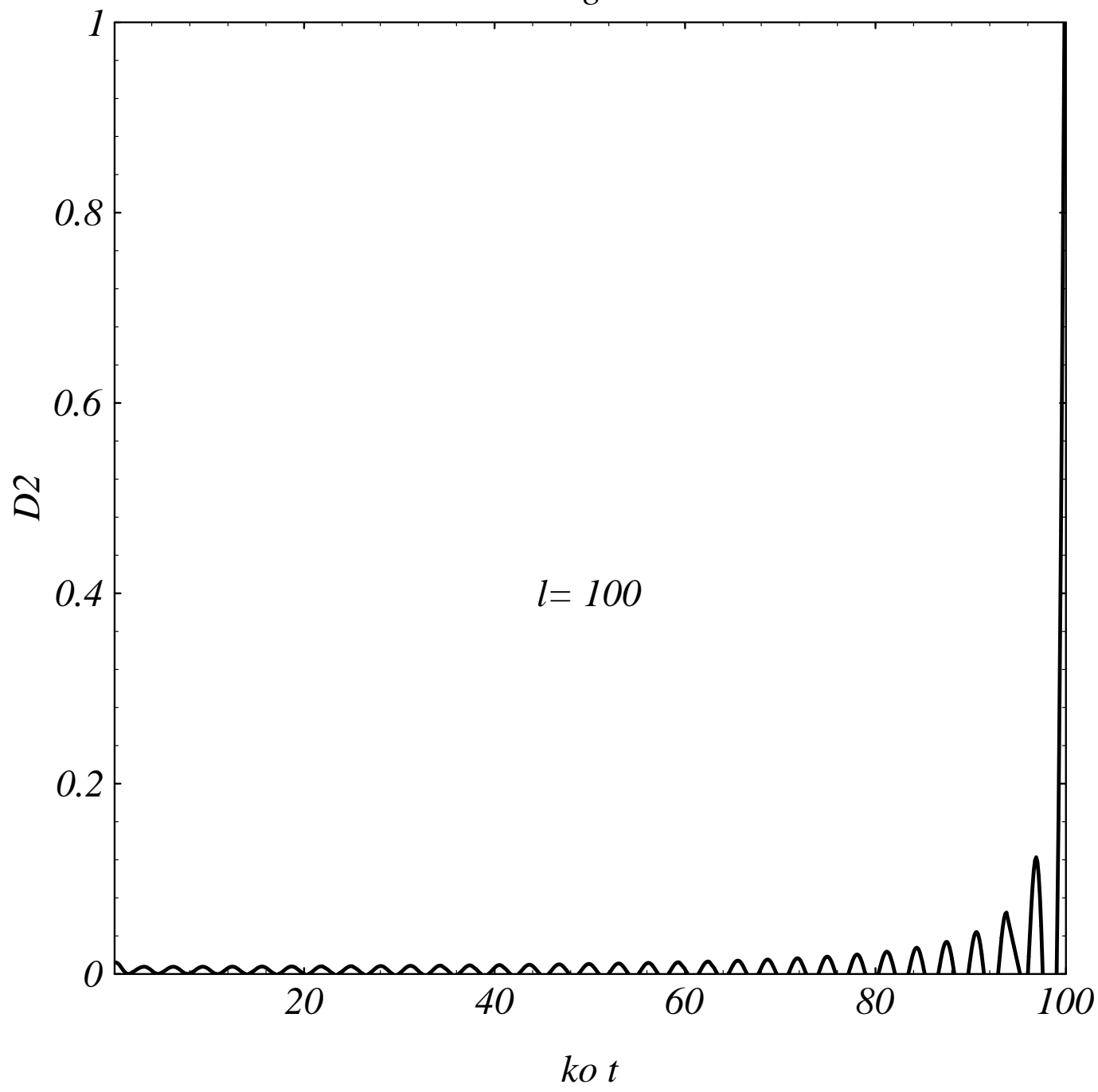


Fig.5

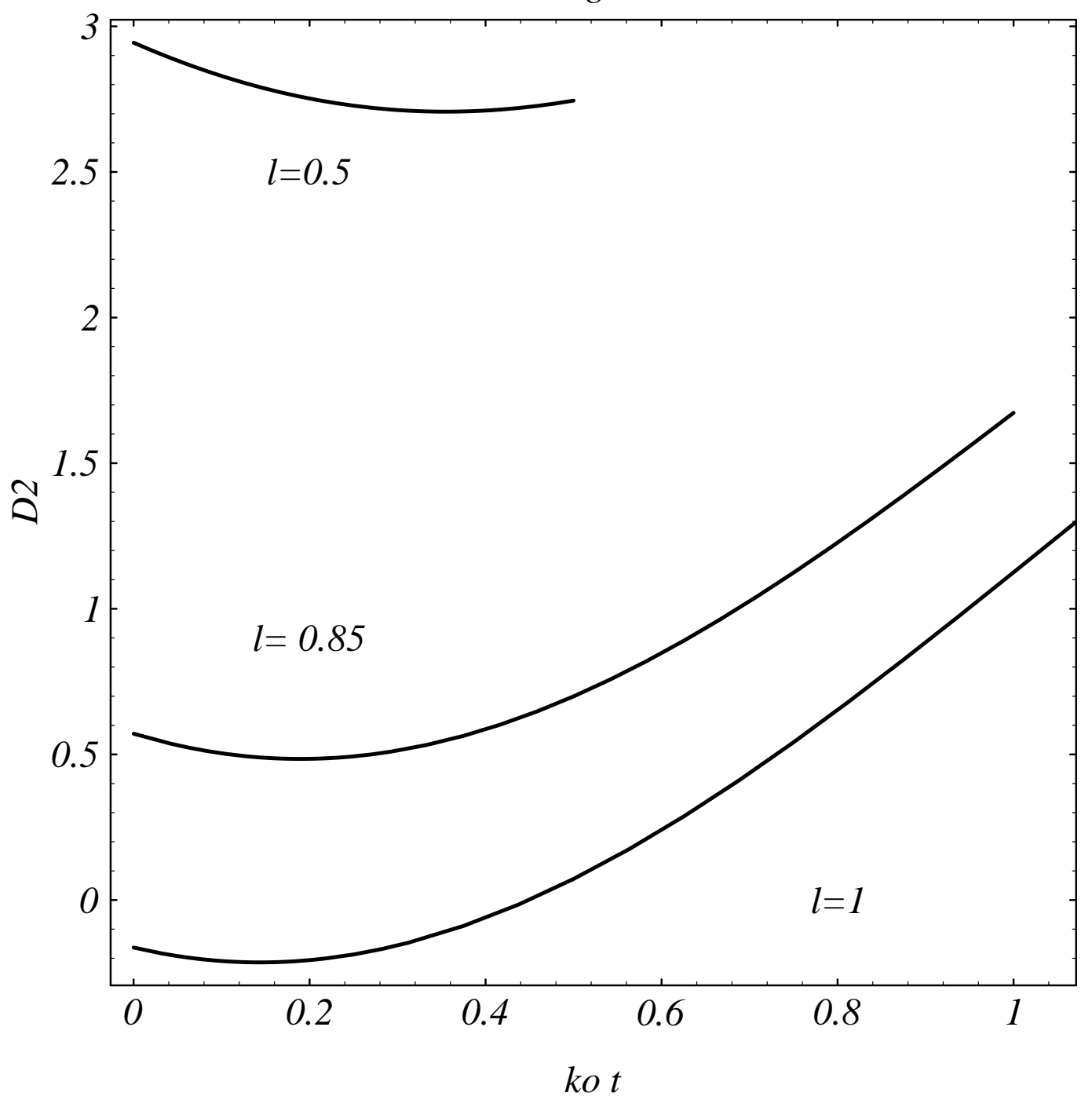

